\title{
Analysis of the direct photon associated spectra from RHIC to LHC
}

D.J Kim*, R. Diaz, T. Alho, Sami Räsänen, J. Rak

Jyväskylä University, Finland

E-mail: djkim@jyu.fi

Direct photon-hadron correlation is a powerful probe of in-medium parton energy loss, i.e the momentum balance dependence of the nuclear modification is expected to be sensitive to the path-length dependence of parton energy loss. In PHENIX the direct photon associated spectra are obtained by a statistical subtraction of the decay photon correlated background so far. We will present the current status of the data analysis in PHENIX and comparisons with PYTHIA and KKP fragmentation function for RHIC and LHC energy as well as the effect of the $k_{T}$ smearing in the spectra.

High-pT Physics at LHC - Tokaj'08

16-19 March 2008

Tokaj, Hungary

${ }^{*}$ Speaker. 


\section{Introduction}

High- $p_{T}$ particle suppression observed in central $A u+A u$ collisions (e.g. [1]) and its absence in $d+A u$ collisions (e.g. [2]) where no excited nuclear medium exists provides quite firm indication of the final state interaction induced by a deconfined QCD medium [3]. Most successful models of partonic energy loss (GLV [4], BDMPS [5], Wang [6] and others) are exploring the inelastically induced gluon radiation process. These models are quite successful in describing the nuclear modification factor $R_{\mathrm{AA}}$ defined as ratio of inclusive particle yield in heavy ion collision to the binary collision scaled yield in $p+p$ data. However, T. Renk and K. Eskola in [7] studied the sensitivity of the $R_{\mathrm{AA}}$ parameter to the details of parton interaction with QCD medium. They argued that due to the strong quenching the particles reaching the detector may be radiated from the jets originating close to the surface of the QCD medium and those produced deep in the medium are fully absorbed. In this case the sensitivity of $R_{\mathrm{AA}}$ to the detailed modeling of the parton energy loss is reduced. In this light it is not surprising that predictions from other models e.g. [8] introducing different mechanism of gluon radiation are also able to reproduce measured values of $R_{\mathrm{AA}}$. It is evident that more complex observables like two-particle correlations or reconstructed jets properties has to be explored.

Another surprise comes from the similarity of $R_{\mathrm{AA}}$ measured for $\pi^{ \pm}$and $p / \bar{p}$ particles [9]. pQCD calculations indicate that a large fraction of $p / \bar{p}$ baryons at high- $p_{T}$ are produced from gluons unlike pions produced more by valence quark interactions. Suppression factors for quark and gluon are expected to by different because of different colored charge $\left(C_{A} / C_{F}=9 / 4\right)$ [10]. However, the measured values of $\pi^{ \pm}$and $p \bar{p} R_{\mathrm{AA}}$ presented in [9] are almost identical in the $p_{T} \geq 5-6 \mathrm{GeV} / c$.

The prompt photon measurement presented by the PHENIX experiment [11] is certainly one of the most interesting results at RHIC. At sufficiently high- $p_{T}$ prompt photons production is dominated by compton scattering $(q+g \rightarrow q+\gamma)$ and to some extent by $q \bar{q}$ annihilation, bremsstrahlung process and quark fragmentation. Photons are blind to the final state interaction and thus no nuclear modification is expected. However, the preliminary data of PHENIX [11] reveals quite significant suppression in the $p_{T} \geq 15 \mathrm{GeV} / c$ region. Although the direct measurement of nuclear shadowing at RHIC energies is not yet available, it is not likely that the suppression of the prompt photon yield by a factor of two at $p_{T} \approx 18 \mathrm{GeV} / c$ could be due to the shadowing [12]. Also the isospin effect (colliding nuclei contain protons and neutrons, however, nuclear data are compared to the yield in $p+p$ and it is known that photo-production cross section in $p+p$ and $n+n$ are slightly different) can explain some part the high- $p_{T}$ suppression, but certainly not all.

It is evident that the measurement of jet fragmentation properties and intrinsic parton transverse momentum $k_{T}$ for $\mathrm{p}+\mathrm{p}$ collisions is vital for the understanding of $\mathrm{pQCD}$ phenomena and it also provides an important baseline for comparison to the results in heavy ion collisions. 


\section{Why Dihadron correlation can't be used for the extraction of Fragmentation Function?}

It has been discussed that one of the unsettled questions related to the high- $p_{T}$ particle quenching is what fraction of energy partons are loosing via the radiative energy loss and what fraction is purely collisional. One of the possible ways to shed more light on this question is to study the modification of the fragmentation function as suggested e.g. in $[13,8]$. The LHC era with yet larger hard-scattering cross section opens the new regime of heavy ion physics allowing to explore the fully reconstructed jets and their properties. However, due to the large particle multiplicity produced in heavy ion collisions the reliable jet reconstruction is possible only at relatively high transverse momenta above $50 \mathrm{GeV}$ or more. In order to explore the "low"- $p_{T}$ region where the jet reconstruction is not possible, the two-particle correlation technique can be used. However, extraction of underlaying parton properties from two-particle correlation functions is not an easy task. For example, it has been believed for decades that the shape of the high- $p_{T}$ trigger hadron associated $x_{\mathrm{E}}$ distributions, where

$$
x_{\mathrm{E}}=-\frac{\vec{p}_{T \mathrm{t}} \cdot \vec{p}_{T \mathrm{a}}}{p_{T \mathrm{t}}^{2}}=-\frac{p_{T \mathrm{a}}}{p_{T \mathrm{t}}} \cos \Delta \phi \simeq-\frac{z_{\mathrm{a}} \hat{p}_{T \mathrm{a}}}{z_{\mathrm{t}} \hat{p}_{T \mathrm{t}}}
$$

and $\hat{p}_{T \mathrm{t}}, \hat{p}_{T \mathrm{a}}, p_{T \mathrm{t}}, p_{T \mathrm{a}}$ are the transverse momenta of the trigger and assocated parton, trigger and associated hadrons and $z_{\mathrm{a}}=p_{T \mathrm{a}} / \hat{p}_{T \mathrm{a}}$ and $z_{\mathrm{t}}=p_{T \mathrm{t}} / \hat{p}_{T \mathrm{t}}$, reflect the shape of the fragmentation function. However, it has been demonstrated in [14] that the fixed momentum of the trigger particle $p_{T \mathrm{t}}$ does not fix the mother parton momentum $\hat{p}_{T \mathrm{t}}$ and it varies with $p_{T \mathrm{a}}$. In this case both $z_{\mathrm{a}}$ and $z_{\mathrm{t}}$ vary with $p_{T \text { a }}$ and this variation completely masks the actual shape of the fragmentation function(illustrated in Figure1).

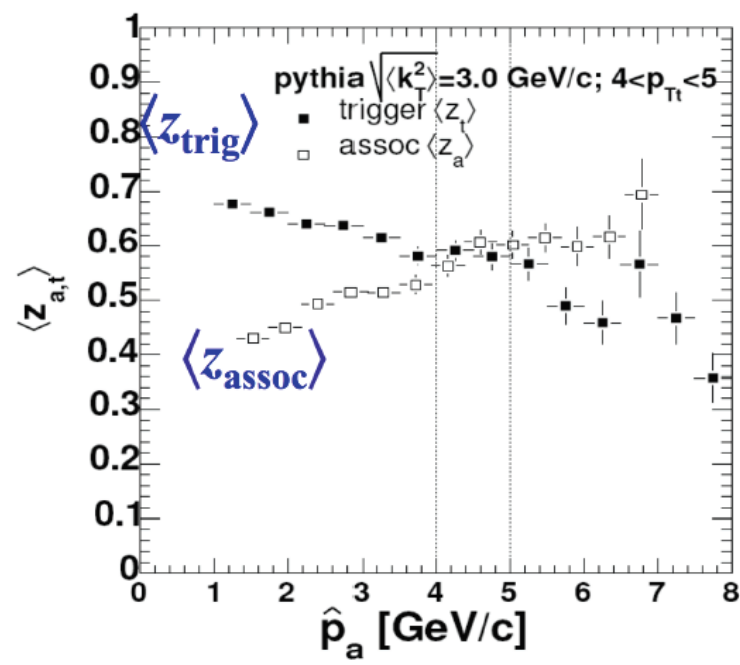

Figure 1: Average $\mathrm{z}$ of a trigger and associated particle as a function of $p_{T \mathrm{a}}$ from PYTHIA simulation shown in [14] 


\section{Gamma Jet Correlations}

One of the alternative ways to explore the fragmentation function is to study the particle distributions associated to the prompt photon. In the high- $p_{T}$ region where the photo-production is dominated by the reverse Compton scattering diagram, the prompt photon balances the back-toback quark. The associated $x_{\mathrm{E}}$ distribution was thought to approximate the fragmentation function of the away side jet. The problem in the method of the previous section is that we cannot constrain the momentum of the trigger parton by constraining the momentum of the trigger particle, which is a fragment of it. If, however, the trigger parton would be a particle that doesn't fragment, the situation would be very different. As discussed in [14], the complicated procedures to solve Eq. (3.1) for the hadrons

$$
\frac{d^{2} \sigma}{d p_{T \mathrm{t}} d x_{\mathrm{E}}}=\frac{d p_{T \mathrm{a}}}{d x_{\mathrm{E}}} \otimes \frac{d^{2} \sigma}{d p_{T \mathrm{t}} d p_{T \mathrm{a}}} \simeq \frac{1}{\hat{x}_{\mathrm{h}}} \int_{x_{T_{\mathrm{t}}}}^{\hat{x}_{\mathrm{h}}\left(p_{T \mathrm{t}} / p_{T \mathrm{a}}\right)} D\left(z_{\mathrm{t}}\right) D\left(\frac{z_{\mathrm{t}} p_{T \mathrm{a}}}{\hat{x}_{\mathrm{h}} p_{T \mathrm{t}}}\right) \Sigma^{\prime}\left(\frac{p_{T \mathrm{t}}}{z_{\mathrm{t}}}\right) d z_{\mathrm{t}}
$$

, in case of direct photon correlation, $D\left(z_{\mathrm{t}}\right) \rightarrow \delta\left(z_{\mathrm{t}}\right)$, can be simplified to Eq. (3.2).

$$
\frac{d^{2} \sigma}{d p_{T \mathrm{t}} d x_{\mathrm{E}}}=\frac{d p_{T \mathrm{a}}}{d x_{\mathrm{E}}} \otimes \frac{d^{2} \sigma}{d p_{T \mathrm{t}} d p_{T \mathrm{a}}} \simeq \frac{1}{\hat{p}_{T \mathrm{a}}} \Sigma^{\prime}\left(\frac{p_{T \mathrm{a}}}{\hat{p}_{T \mathrm{a}}}\right) D\left(\frac{p_{T \mathrm{a}}}{\hat{p}_{T \mathrm{a}}}\right)
$$

A high- $p_{T}$ photon emerging from a hard $\mathrm{pQCD}$ interaction would serve exactly such a purpose, and since it only interacts electromagnetically, it would have the added advantage of surviving relatively unperturbed through a strongly interacting medium in Heavy Ion collisions.

This method is called gamma-tagging, and was originally proposed in [15]. However, also in this case there are important effects which need to be taken into account. One constraint comes from the fact that at low $p_{T}$ the prompt photon production may be contaminated by fragmentation photons from the large number of gluonic jets produced in heavy ion collision [16]. Fragmentation photons obviously do not balance the away-side parton.

Another constraint comes from the fact that even when considering the leading order Compton diagram there is always soft QCD radiation which breaks the jet-photon momentum balance and the azimuthal collinearity. As a result of soft QCD radiation, this non-perturbative radiation manifests itself as a non-zero value of the net parton-photon pair transverse momentum magnitude $p_{T \text { pair }}$. Originally this soft radiation induced transverse momentum was attributed to the individual incoming partons and the notation of $k_{T}$ (parton transverse momentum) was introduced by Feynman, Field and Fox [17], where the colliding partons might have some initial transverse momentum $k_{T}$ with respect to the incoming hadrons. This analog of the Fermi motion of nucleons in a nucleus would give rise to a smearing out of the $p_{T}$ spectrum. There could be an additional smearing due to the $k_{T}$ associated with the fragmentation in the final state [18].

As discussed in [14] the $k_{T}$ may be viewed as a sum of three different contributions:

- Intrinsic parton Fermi-momentum [17].

- Soft QCD radiation [19].

- Hard NLO radiation. 


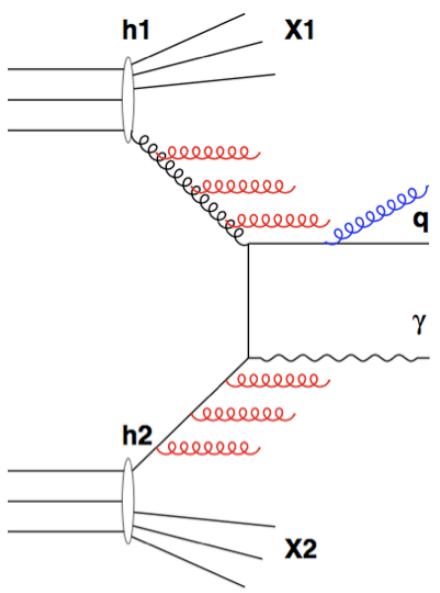

(a)

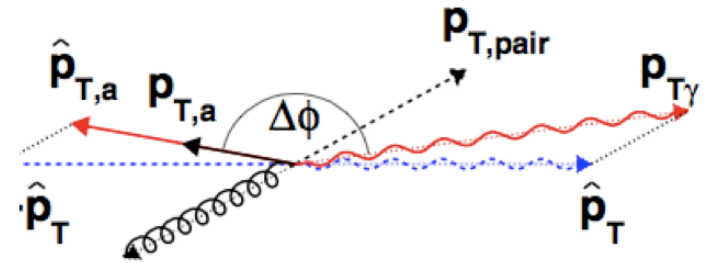

(b)

Figure 2: (a) QCD compton scattering $=($ Soft + hard QCD radiation) (b) Schematic view of Compton $q+g \rightarrow$ $q+\gamma$ photo-production event in the plane per perpendicular to the beam. In purely leading order and in the center of mass of the hard scattering, the outgoing photon and quark (blue arrows) are exactly back-to-back. The soft QCD radiation, represented by single gluon line, leads to the accoplanarity and imbalance between the photon, $p_{T \mathrm{t} \gamma}$ and quark, $p_{\hat{T} \mathrm{a}}$

One of the good reaction to study this transverse momentum effects is high-mass lepton pair production. The lowest order subprocess is $q \bar{q} \rightarrow l^{+} l^{-}$. If only this subprocess is used, the transverse momentum of the lepton pair gives a direct measure of the intrinsic parton transverse momentum. For example, data at $\sqrt{s}=27.4 \mathrm{GeV}$ indicate $\left\langle p_{T}^{2}\right\rangle=1.9 \mathrm{GeV} / \mathrm{c}^{2}$. This corresponds to an intrinsic $<k_{T}^{2}>$ of $0.95 \mathrm{GeV}^{2}$ or, assuming a gaussian shape; $<k_{T}>=864 \mathrm{MeV}$. PHENIX also measured this quantity via two particle correlation method at $\sqrt{s}=200 \mathrm{GeV}$ which shows the larger value of $k_{T}$ than expected [14]. In addition to the fact that there are still theoretical problems in defining $k_{T}$, there are also ambiguities in how one incorporates the distribution into a specific calculation. We have showed the derivation of formula published in [14] based on the following logic: we first considered the back-to-back pair $\hat{p_{T}},-\hat{p_{T}}$ and evaluated the boost according the random $\hat{p}_{\text {pair }}$ vector which has a uniform angular distribution ( see Fig 2 (b) ) with respect to the $\hat{p_{T}}$ and its magnitude corresponds to the $2 \mathrm{D}$ Gaussian distribution of variance $\sqrt{<2 k_{T}^{2}>}$. This leads us to have a very simplified formula, which is not discussed here but will be published in other paper later. One of the results from this approach is shown in this paper and the effect of $k_{T}$ smearing will be discussed in latter sections. 


\section{1 $\sqrt{\left\langle k_{T}^{2}\right\rangle}$ at RHIC and LHC}

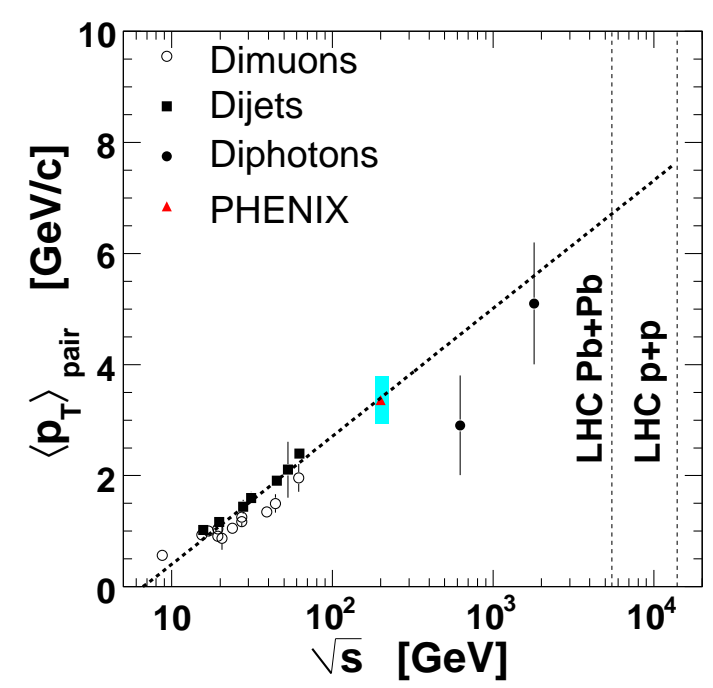

Figure 3: The net dimuon (open circles), dijet (solid squares), diphoton (solid circles) and back-to-back partons (red triangle) transverse momenta measured at various center of mass energies (see [20] and [14]). PHENIX back-to-back parton value is derived from dihadron accoplanarity when the average trigger hadron momentum fraction $z_{\mathrm{t}}$ is taken into account.

The magnitude of Fermi-momentum (truly intrinsic parton momentum) is determined by the transverse size of colliding nucleons and it is of order of $300 \mathrm{MeV} / \mathrm{c}$. The large values of $\sqrt{\left\langle k_{T}^{2}\right\rangle} \approx$ $3 \mathrm{GeV} / c$ at RHIC indicates that the soft and hard radiation are the dominant contributions. It evidently implies that it would be more appropriate to talk about the net pair transverse momentum rather than about $k_{T}$ attributed to individual partons. However, we will stick to the historical notation of Feynman ${ }^{1}$ et al..

The net back-to-back parton transverse momentum $p_{T \text { pair }}$ induced by three different contributions discussed above will lead to the imbalance of the prompt photon and quark energies. One way of overcoming the constraint is to trigger on photon momenta much larger than the actual value of $k_{T}$. Extrapolation from measured values of $\sqrt{\left\langle k_{T}^{2}\right\rangle}$ to LHC energy regime

$$
\left\langle p_{T}\right\rangle_{\text {pair }} \approx \log (0.15 \cdot \sqrt{s})
$$

(see Fig. 3) leads to the values larger by factor of two $\left(\left\langle p_{T}\right\rangle_{\text {pair }}=7.7 \mathrm{GeV} / \mathrm{c}\right.$ and $\sqrt{\left\langle k_{T}^{2}\right\rangle}=6.1 \mathrm{GeV} / \mathrm{c}$ at $\sqrt{s}=14 \mathrm{TeV}$ ) as compared to RHIC. If this will be the case at LHC energy then for the unbiased region of prompt photon momenta where the $\left\langle p_{T}\right\rangle_{\text {pair }}$ will be negligible lies probably way above $p_{T \mathrm{t}} \geq 20 \mathrm{GeV} / c$.

\footnotetext{
${ }^{1}$ in the case of $2 \mathrm{D}$ Gaussian distribution there is a trivial realtion $\left\langle k_{T}^{2}\right\rangle=\frac{\pi}{2}\left\langle p_{T}\right\rangle_{\text {pair }}$
} 


\subsection{Results at RHIC and Simulations at LHC}

At RHIC energy, the identification of direct photons is difficult due to the large number of background photons from hadronic decays, mostly from $\pi^{0}$ decays. Therefore the extraction of the direct photon-hadron pairs per trigger yields relies on a statistical subtraction of the decay photonhadron per trigger yields from the inclusive photon-hadron pairs per trigger yields. The first result from this subtraction method is shown in Fig. 4. The direct photon-hadron yield per trigger yield in near-side is consistent with zero, which is seen in PYTHIA simulation. Also the away-side yields are comparable with PYTHIA.
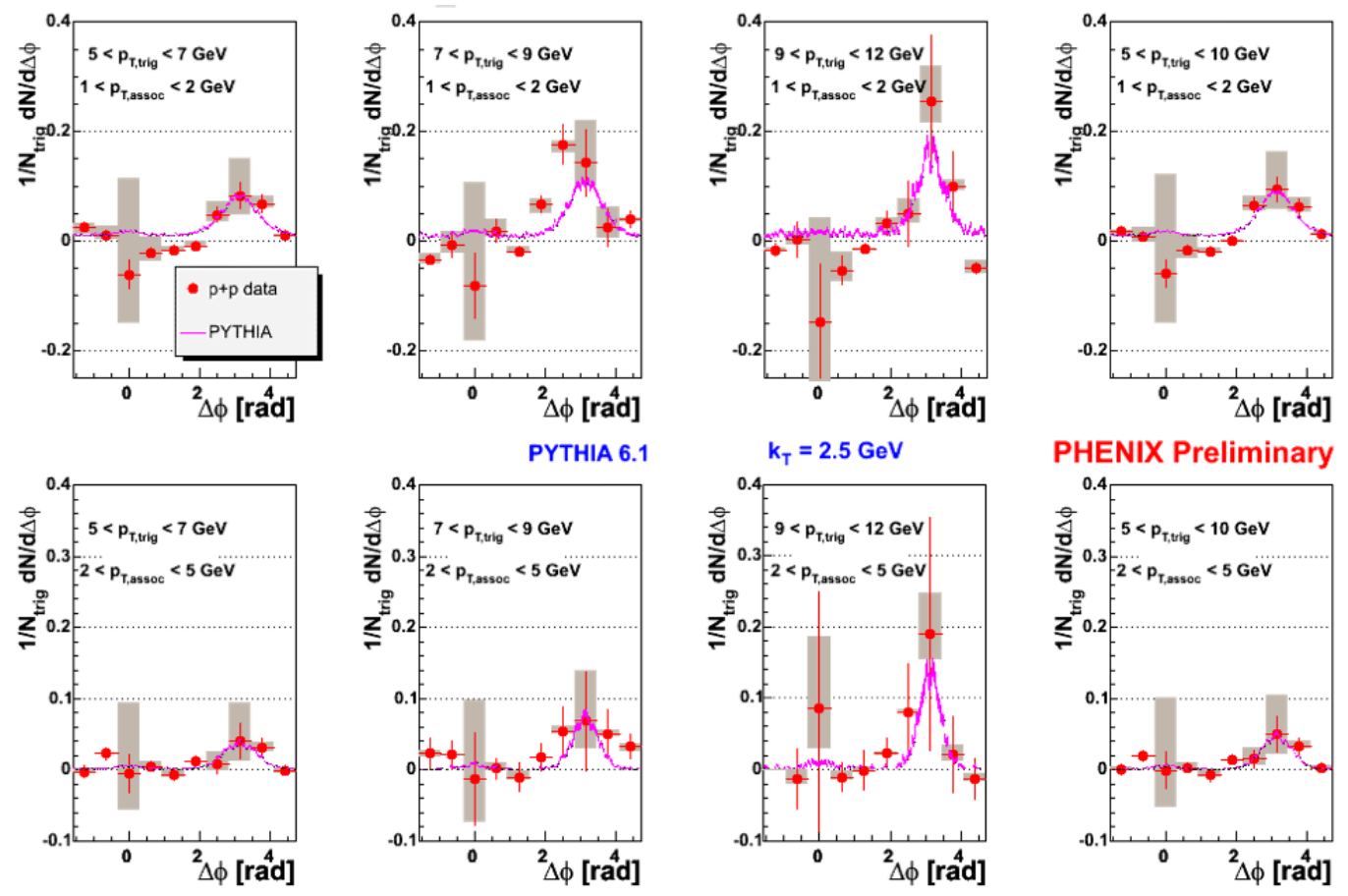

Figure 4: Comparison of PYTHIA correlation functions with PHENIX Preliminary data in $p+p$ at $\sqrt{s}=200$ $\mathrm{GeV}$.

In the Figure 5, PYTHIA $x_{E}$ distributions for quark and gluon are fitted with KKP fragmentation function. The distribution of associated charged particles with $x_{\mathrm{E}}$ variable, equation 2.1, for various quark or gluon trigger $p_{T t}$, results of KKP fragmentation $[14,21]$ fits are shown as dashed lines. PYTHIA agrees well with KKP but there seems to be different in low $p_{T}$ trigger bins. As it was demonstrated in [14] and it is shown again in Figure 6 for $\pi^{0}$ hadron correlations in PYTHIA, the $x_{\mathrm{E}}$ Slopes are getting bigger as the trigger $p_{T}$ grows both for RHIC and LHC energies. We conclude again from this study that the fragmentation function is not accessible in $\pi^{0}$-hadron correlations because varying $p_{T a}$ for fixed $p_{T t}$ leads to variation of both trigger and associated jet energies.

In order to evaluate the feasibility to extract the quark and gluon fragmentation function from the $x_{E}$ distribution we compared the negative slope of the exponential fit with the negative inverse logarithmic derivative of the quark and gluons fragmentation function into charged hadrons ex- 

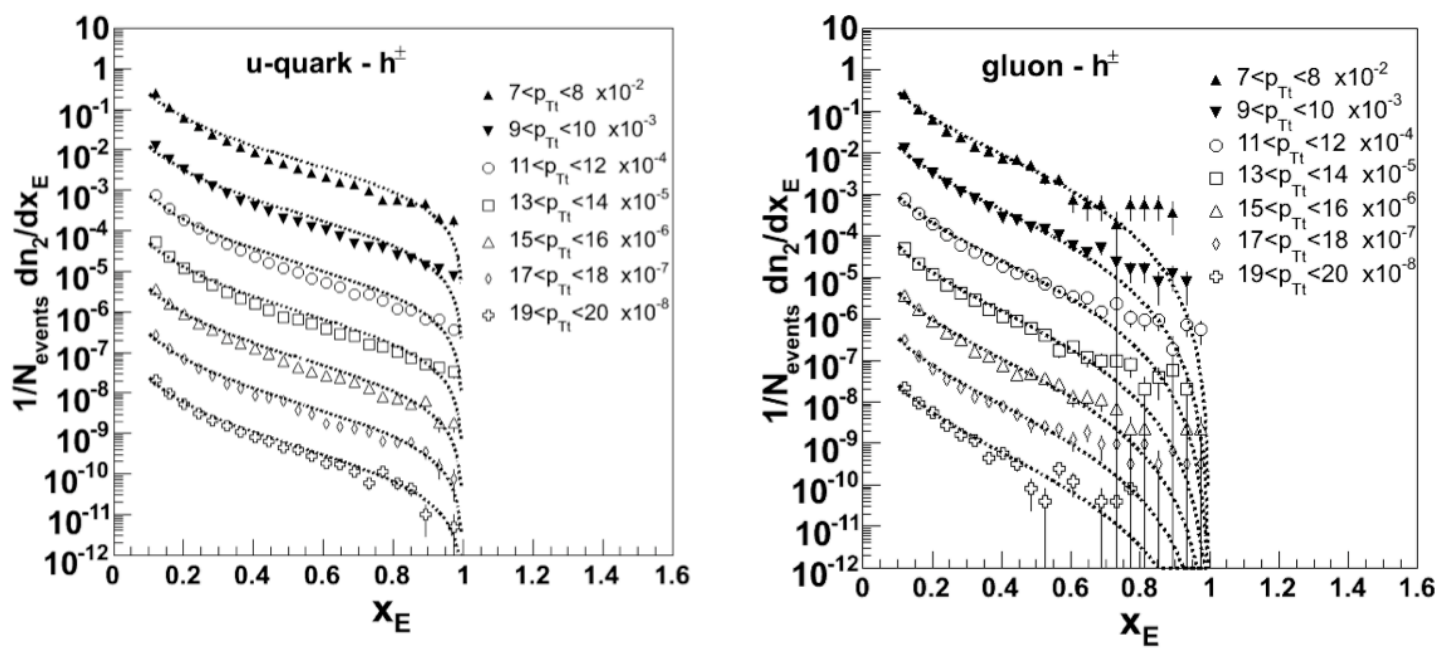

Figure 5: PYTHIA $x_{E}$ distributions ( quark(left), gluon(right) ) are fitted with KKP fragmentation function. The distribution of associated charged particles with $x_{\mathrm{E}}$ variable, equation 2.1, for various quark or gluon trigger $p_{T t}$, results of KKP fragmentation $[14,21]$ fits are shown as dashed lines. The intrinsic $k_{T}$ value at LHC was extrapolated to $5 \mathrm{GeV} / \mathrm{c}$

tracted from the KKP parametrization $[14,21]$ in the Figure 5. The very interesting feature is shown in case of $\gamma-h$ that in low $p_{T}$ trigger the $x_{\mathrm{E}}$ slopes are still increasing as the trigger momentum gets larger and only at relatively high trigger-photon momentum the slopes are getting flatter and closer to the KKP quark fragmentation function. This is shown in PHENIX data and PYTHIA as well. Increasing trends are similar as $\pi^{0}$-hadron and even $\gamma-h$ is much steeper with the same trigger bin.

In order to understand this behavior along with $k_{T}$ effect in low $p_{T}$ region, we have revisited the method of Lorentz invariant $k_{T}$ smearing discussed in (sub- section B. $k_{T}$ smearing in section VI. DIJET FRAGMENTATION [14]). We found a lot simpler analytical solution to the problem discussed in the paper. The new formalism is used to compute a direct photon or high- $p_{T} \pi^{0}$ associated $x_{\mathrm{E}}$ distributions. With this new formula, a conditional probability for detecting photon $p_{T t}$ and associated $p_{T a}$ given parton momentum $p_{T}$ in CMS of hard scattering( see Figure 2 (b) ) is calculated and shown in Figure 7 for RHIC(a) and LHC(b). The conditional probability for given parton $p_{T}$ is smeared a lot by $k_{T}$ in low $p_{T}$ and washed out in higher $p_{T}$. At RHIC energy with the photon trigger $8 \mathrm{GeV}$ the distribution is still distorted from gaussian shape shown in the bottom of Figure 7 (a).

Taking into account that Compton scattering is the dominant process to the direct photon production in $p+p$ collisions, is possible to extract the quark fragmentation function from direct photons-jet correlations, without correction of the $k_{T}$ smearing, only at relatively high triggerphoton momentum where the $k_{T}$ bias is negligible and the jet and photon energy are balanced. 


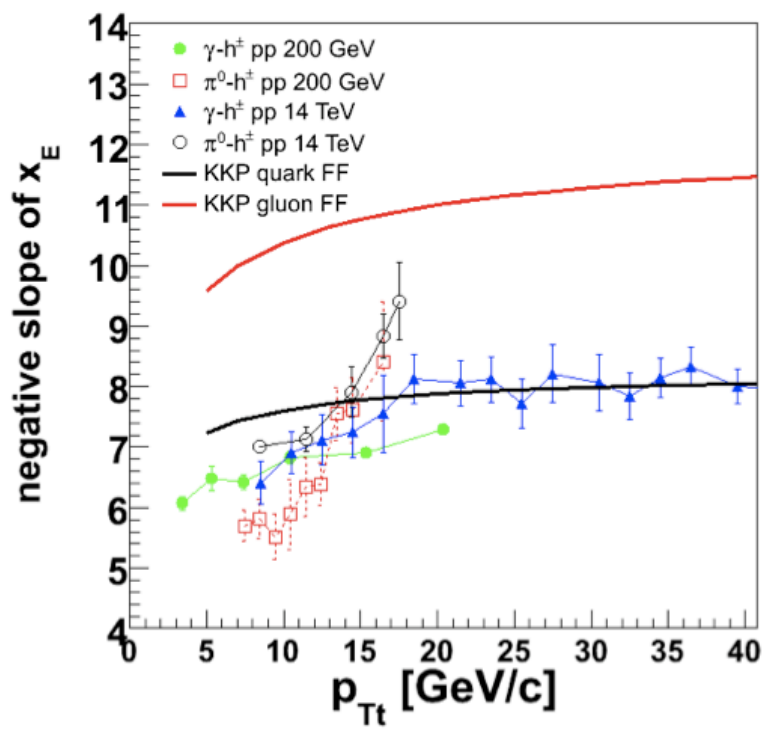

Figure 6: PYTHIA and KKP comparison, the negative slope parameter extracted from the fit of an exponential function to the $x_{\mathrm{E}}$ distributions in different cases. The slopes are compared with the negative logarithmic derivative of quarks and gluons fragmentation function from the KKP parameterization [14, 21] .

(a)

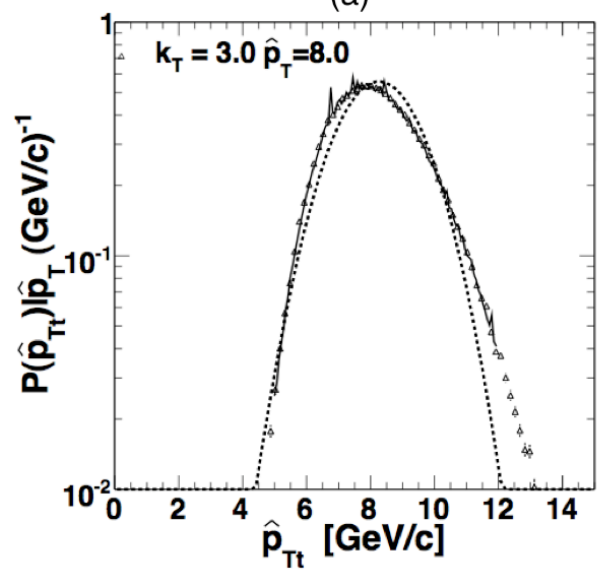

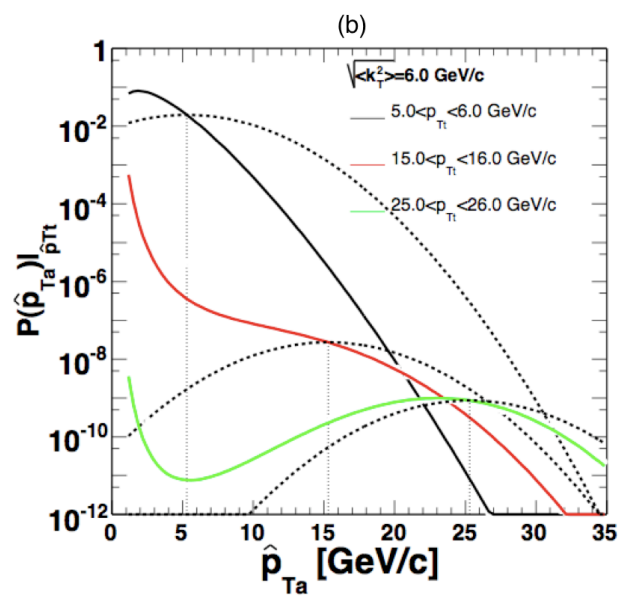

Figure 7: Conditional probability for detecting photon $p_{T t}$ and associated $p_{T a}$ given parton momentum $p_{T}$ in CMS of hard scattering( see Figure 2 (b) ) for RHIC(a) and LHC case(b). 


\section{Conclusions}

The jet fragmentation variable $z=p_{T a} / E_{\text {jet }}$ can be extracted from the $x_{E}$ variable defined in Equation 2.1, where $p_{T a}$ is the associated hadron transverse momentum from the jet with energy $E_{j e t}$ and $p_{T t}$ the transverse momentum of the trigger particle. In the case of $\gamma$-jet correlations the $p_{T t}$ quantity is replaced by the photon momentum that should be equal to the jet energy in absence of the $k_{T}$ effect. Therefore the away-side charged particle distribution associated to the direct photon should scales with the jet inclusive fragmentation function into charged particles. But from our recent studies described in the previous section, it is evident that at LHC one can expect that the imbalance due to the $k_{T}$ smearing completely washes out the correlation between the quark and photon energies for the photon energy below $10 \mathrm{GeV} / c$ and the distributions deviate from the Gaussian function quite significantly even at $p_{T \gamma}=15 \mathrm{GeV} / c$. In order to extract the fragmentation function from the prompt photon associated distributions in the $p_{T \gamma}$ region below 30 $\mathrm{GeV} / c$ at LHC the detailed understanding of $k_{T}$ phenomena is necessary. PHENIX Preliminary data show the expected trends compared with our $k_{T}$ smearing formula and PYTHIA but more higher precision data with larger $p_{T}$ trigger bins will be needed to verify the trigger bias and $k_{T}$ effect quantitatively. 


\section{References}

[1] PHENIX, S. S. Adler et al., Phys. Rev. Lett. 96, 202301 (2006), nucl-ex/0601037.

[2] PHENIX, S. S. Adler et al., Phys. Rev. Lett. 98, 172302 (2007), nucl-ex/0610036.

[3] PHENIX, K. Adcox et al., Nucl. Phys. A757, 184 (2005), nucl-ex/0410003.

[4] M. Gyulassy, P. Levai, and I. Vitev, Phys. Rev. Lett. 85, 5535 (2000), nucl-th/0005032.

[5] R. Baier, Y. L. Dokshitzer, A. H. Mueller, S. Peigne, and D. Schiff, Nucl. Phys. B484, 265 (1997), hep-ph/9608322.

[6] Q. Wang and X.-n. Wang, Phys. Rev. C71, 014903 (2005), nucl-th/0410049.

[7] T. Renk and K. J. Eskola, (2006), hep-ph/0610059.

[8] N. Borghini and U. A. Wiedemann, (2005), hep-ph/0506218.

[9] STAR, B. I. Abelev et al., Phys. Rev. Lett. 97, 152301 (2006), nucl-ex/0606003.

[10] STAR, B. I. Abelev et al., (2007), nucl-ex/0703040.

[11] PHENIX, T. Isobe, (2007), nucl-ex/0701040.

[12] K. J. Eskola, V. J. Kolhinen, and R. Vogt, Nucl. Phys. A696, 729 (2001), hep-ph/0104124.

[13] X.-f. Guo and X.-N. Wang, Phys. Rev. Lett. 85, 3591 (2000), hep-ph/0005044.

[14] PHENIX, S. S. Adler et al., Phys. Rev. D D74, 072002 (2006), hep-ex/0605039.

[15] I. S. Xin-Nian Wang, Zheng Huang, arXiv:hep-ph/9605213v1 (1996).

[16] F. Arleo, (2007), hep-ph/0701207.

[17] R. P. Feynman, R. D. Field, and G. C. Fox, Phys. Rev. D18, 3320 (1978).

[18] J. F. Owens, Rev. Mod. Phys. 59, 465 (1987).

[19] A. Kulesza, G. Sterman, and W. Vogelsang, Nucl. Phys. A721, 591 (2003), hep-ph/0302121.

[20] L. Apanasevich et al., Phys. Rev. D59, 074007 (1999), hep-ph/9808467.

[21] B. A. Kniehl, G. Kramer, and B. Potter, Nucl. Phys. B597, 337 (2001), hep-ph/0011155. 